\title{
Rise of Crypto Currency-Bitcoin and Impact on the Financial System
}

\author{
G. Taviti Naidu; Muyinda Gerald Dhatemwa
}

\begin{abstract}
A product of the $21^{\text {st }}$ century, crypto-currency is a digital representation of a value that can be digitally traded and functions as a medium of exchange, a unit of account or store of value. Safety, simplicity, affordability, reachability, and transparency are advantages however, challenges such as fraud, complexity, money laundering plague Bitcoin, Lite coin, Dodge coin, Amazon coin,ethereum coin, Nemcoin, Libra, DC/EP coin.

Key Words: Crypto-Currency, Bitcoin, Financial system
\end{abstract}

\section{I.INTRODUCTION}

Money has been in existence for the last 3000 years originating from China as rudimentary coins to being officially minted in Lydia by king Alyattes 600B.C.It has acted as a means of exchange and store of value since the $15^{\text {th }}$ century replacing the barter system which involved trading "something for something", this not only took a lot of time but was also cumbersome. 700B.C witnessed the rise of paper money in China and was eventually adopted by England and Europe as a whole in 1666, its rise improved financial stability and confidence not only in domestic trade but more so international trade leading to the rise of strong financial systems in England, Europe and Asia however, challenges such as hyperinflation and counterfeiting resulted in countries such as China temporarily abandoning paper money in $1455.21^{\text {st }}$ century gave rise to electronic payments through the invention of the internet in 1969 by ARPNET a USA military project and taken advantage of by Stanford Federal Credit Union in 1994 which introduced online payments and online banking system to its members later spreading to other financial institutions leading to rising to EFTs, credit cards, debit cards, prepaid cards and eventually E-cash of Cyber coin in 1966. The concept had been introduced by computer engineer Wei Dai ten years before cryptocurrency. In 1998 he released a paper about "Bmoney," the concept of digital currencies that could be sent along with a collection of cryptographic pseudonyms that cannot be traced. The same year, however, another attempt by the name of Bit Gold was introduced on 31 October 2008 by blockchain founder Nick Szabo, the idea was ultimately perfected by Satoshi Nakamoto who piloted the first developed block, Genesis. Virtual currency is not bound by borders, minimal regulation and much convenient in the $21^{\text {st }}$ century due to their adaptability to the internet and modern technology, 2020 witnessed the rise of over 2000 virtual currencies globally valued at 237.1 billion dollars.

Revised Manuscript Received on October 10, 2020.

* Correspondence Author

Dr. G. Taviti Naidu*, MBA, M.Phil, Ph.D., Post Doctoral Fellow, ICSSR (Andhra University) Visakhapatnam. E-mail: dr.tavitinaidugongada@gmail.com

Muyinda Gerald Dhatemwa, M.B.A student, faculty of Finance, Andhra University, Visakhapatnam, India. E-mail: dhatemwag@gmail.com

(C) The Authors. Published by Blue Eyes Intelligence Engineering and Sciences Publication (BEIESP). This is an open access article under the CC BY-NC-ND license (http://creativecommons.org/licenses/by-nc-nd/4.0/)
However, unlike few countries like Switzerland that have openly accepted virtual currency such as Crypto,many countries globally have either banned (China, Bulgaria) virtual currencies in the form of crypto or refused to formally recognize them as official currencies. (India, Netherlands). Rejection of Crypto-currency in countries such as China isfear of anonymity, money laundering, illicit trading, and tax avoidance. These negative factors are all against the traditional motives of the invention of "money" and until ably addressed by the users, skepticism and negative regulation will surround cryptocurrency. By 2019, cryptocurrency scam in form of Bitcoin scams amounted to more than 4billion US dollars from players such as; One coin, Plexcoin, Bit connect, Pincoin, and ifan. Therefore, the researcher tried to exploit factors of cryptocurrency and its impact on the financial system.

\section{II.CRYPTO-CURRENCY - THE CONCEPT}

Bill Gates founder of Microsoft likened Bitcoin to a "techno tour de force", modernity and speed at which it's changed the financial system has been unforeseen, positioning many governments and regulators playing catch-up in terms of regulation and control. Unfortunately, advantages of anonymity, profitability and exchanging value without regulation from an institution have equally drawn negative attention and criticism from financial regulators, Jamie Dimon Chairman and CEO;JP Morgan Chase one of the largest banks in the USA,referred to Bitcoin as a "bubble worse than tulip bulbs, a fraud in the making that would not end well"Such polarizing statements made by two of the most influential global personalities have created uncertainty and intrigue around Bitcoin but also virtual currencies as a whole. Virtual currency maintains features of traditional currencies based on an idea of exchanging value without regulation or approval of an institution however having no legal tender. According to the European Central Bank, Virtual currencies are unregulated digital money issued and controlled by its developers and accepted in a specific virtual community however later on in 2015,the European Central Bank later eliminated the term "money" as they lack a high liquid asset and lack of public acceptance and the term "unregulated" as a few European countries had adopted guidelines and regulations. Virtual currencies can thus be defined as a digital representation of value not issued by a credit institution or central bank which in some cases can be used as an alternative money. Cryptocurrency is based on two technical solutions; "blockchain and mining," the blockchain is part of the electronic framework of

Published By:

Blue Eyes Intelligence Engineering \& Sciences Publication

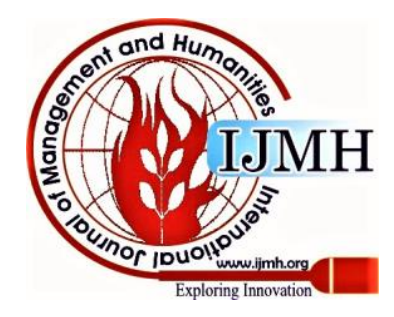


Bitcoin which serves as the accounting mechanism of the network using a 'Distributed Ledger Technology' (DLT) is a scheme that allows for 'a database of globally distributed mirrored, linked, which coordinated digital records.This tracks recent transactions as blocks and connects them to previous transactions, maintains a historical overview of all network-wide operation, provides a comprehensive archive of all Bitcoin transactions ever created which is completely available, which users depend on similar versions of the ledger - the database is stored on several network-wide servers, or 'nodes,'.The Blockchain, as an accounting tool for a distributed network, represents a significant breakthrough in the double-entry bookkeeping processes that central banks and the traditional financial system have depended on for decades. Blockchains' protection approach was to classify users on the blockchain, not by my name but by alphanumeric 'shared keys' linked with their account, a function that many financial intuitions and governments envied and equally adored. Bitcoin's value is generated by mining that provides consumers with an opportunity to engage in which a transaction is sent to the Bitcoin network. A member in the network must verify the transaction before the transaction is registered on the Block Chain and merge it into a block with other pending transactions, computers in the network then rush to determine, or 'mine,' a solution to a cryptographic problem that enables the block to be connected to a previously verified block. When a miner discovers a solution, other network members verify the consistency of their work ensuring the validity and can be added to the blockchain helping to protect the Bitcoin network and requiring the blockchain to serve as an eternal database, while changing the transactional information contained on the blockchain would entail replication of the entire mining cycle from the period Bitcoin was established - an inference

\section{III.FINANCIAL DISRUPTIONS OF CRYPTOCURRENCIES:}

Anonymity, Bitcoin uses an alphanumeric code that does not take into account the conventional financial way of marking a transaction with the customer's name and address, besides, a "mixing" strategy that enables Bitcoin users to transfer Bitcoin's from one address to a mixing service and receive Bitcoin's mixing service to another address that could not be connected to the original one, operated by a central authority. To count as a legal transaction, all users involved in the mixing, blind signatures will sign the transaction ensuring that the meeting server does not know details about the linkability between the input and the output address.However, the likelihood that the mixer might deanonymize users using its encrypted information is still high scenarios such as "silk road" operated by Ross Ulbricht, who used bitcoin as a big payment, enabling one of the biggest electronic drug exchange valued at \$4 billion, while it was a wake-up call for many policymakers to either strictly control Bitcoin or solely ban in the case of China.

Figure-1:Bitcoin mixing

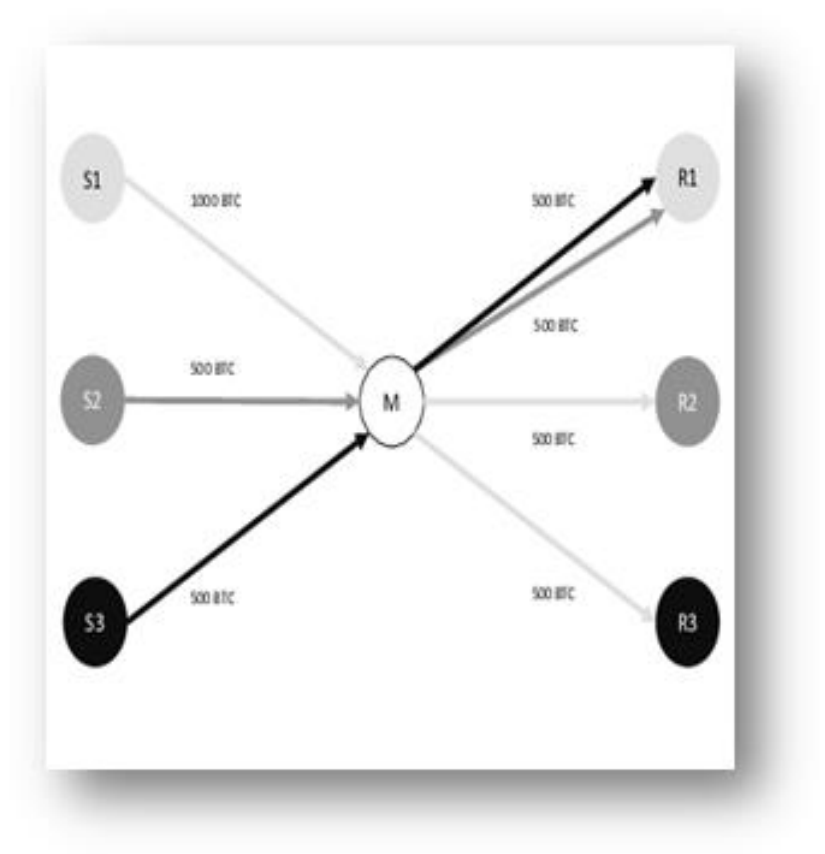

\section{[1] www.researchgate.net/figure/Bitcoin-mixing- example_fig1_322914722}

Interpretation:Accessibility and convenience, Bitcoinis based on decentralized autonomous cooperation (DAC), a form of cooperation that is code on the cloud-based algorithm set up to perform certain characteristics that create value. DAC is based on autonomous technology that is engrossed in today's modern devices making it easily reachable and accessible to users through phones and computers, such innovation has created stiff competition between traditional banks whose transactions take longer with unnecessary red tape also, users of Bitcoin don't need to walk into offices and fill documents rather they act remotely on mobile devices encouraging a few banks to study the DAC system but also revamp their traditional banking systems with features such as e-banking.

Figure-2:Transactions "before" and "after" Bitcoin

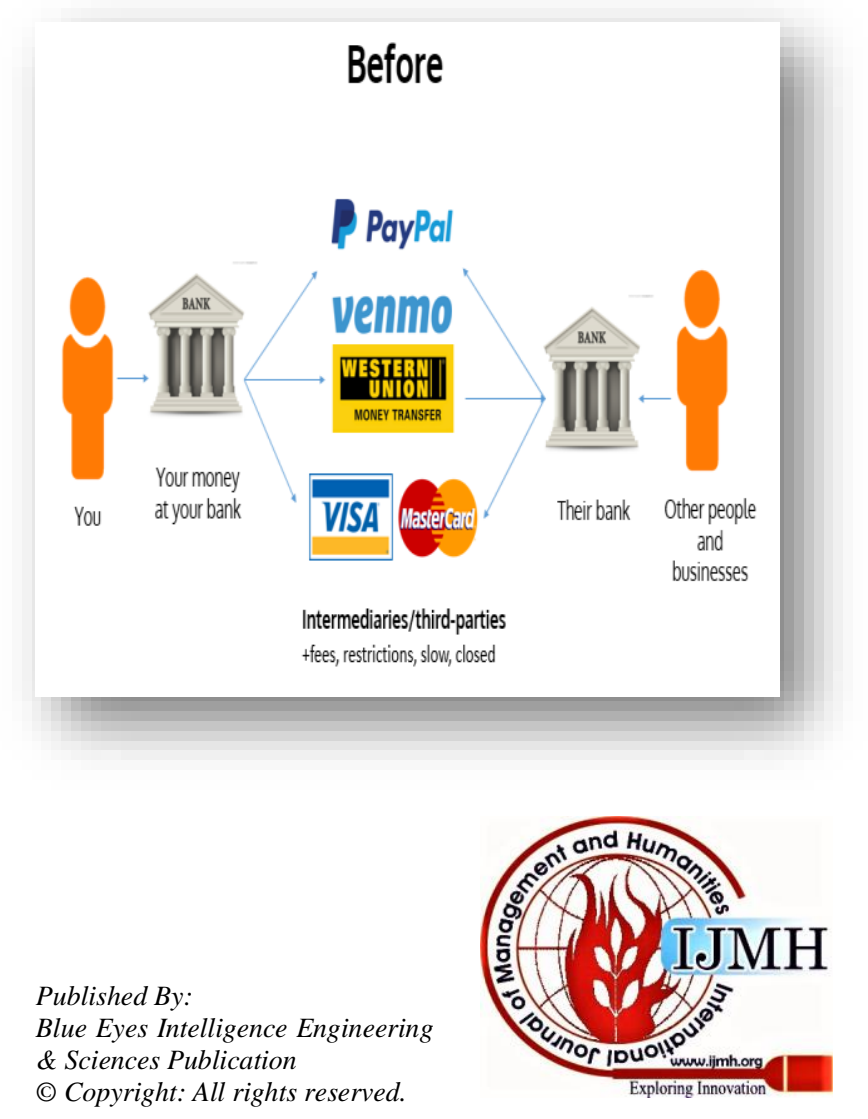




\section{After w/ Bitcoin}

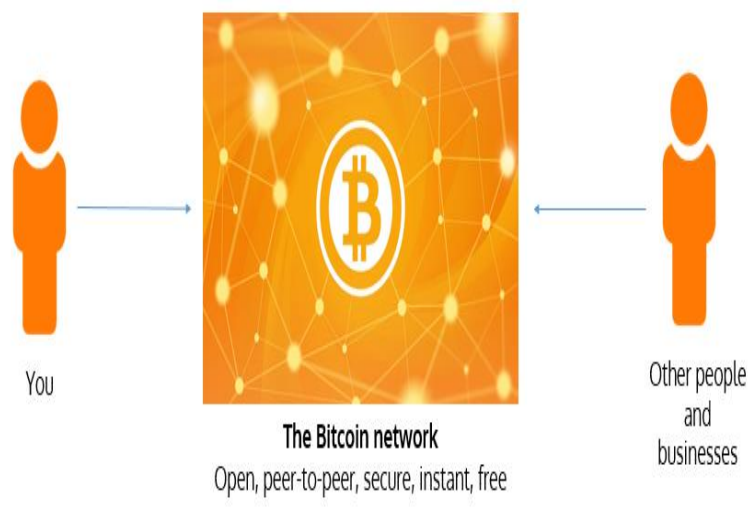

[2]. www.medium.com/@liamzebedee/3-essentialtakeaways-from-the-mit-microsoft-Bitcoin-talk54a4cd71a702\#.17zeppbil

Interpretation: Digital scarcity, Bitcoin relies on algorithms that create artificial scarcity controlling demand and supply as banks regulate money supply in the financial system. The scarcity of the Bitcoin is determined by the difficulty in "mining" and value-driven from the present and future supply giving an incentive to the owners of Bitcoin to purchase Bitcoin employing intensive computer machinery to solve complicated algorithms however this creates abnormal power consumption estimated to consume as much power as Denmark. However,by 2030Bitcoin will approach their optimum of 21 millionand according to programming protocols,no programmer currently can manipulate this feature making it vulnerable to skepticism about finite digital scarcity, unlike traditional currency.

Figure-3: Total Bitcoins in circulation over time (millions)

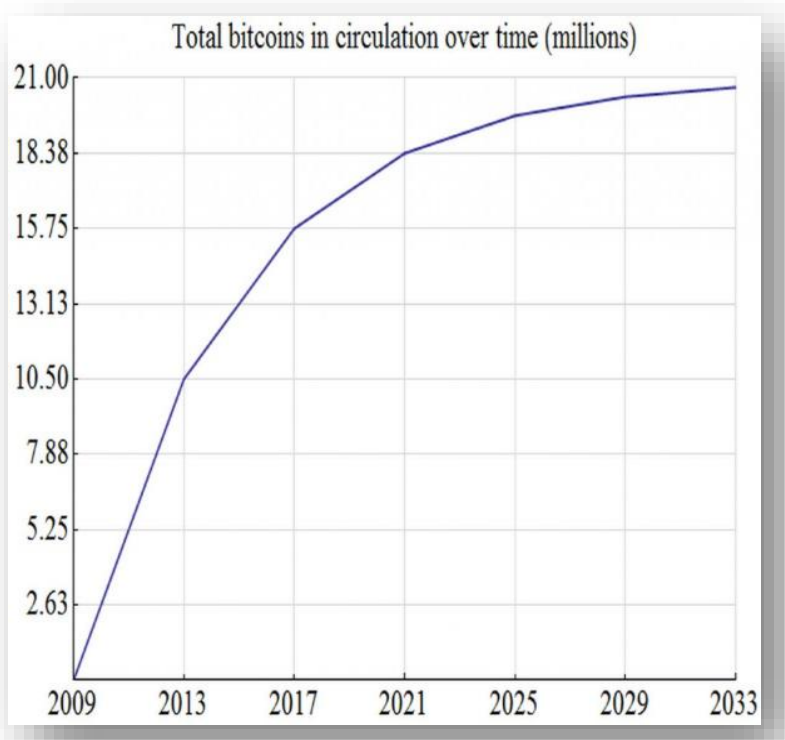

[3]. www.vamos.ee/easy-trade/limited-supply-of-Bitcoinethereum-mh/
Interpretation: Transparency, unlike the traditional banking system the Bitcoin system is based on decentralized trust which requires the user to be able to track an initial transaction from the point of being accepted by the Bitcoin mechanism to finally being recorded in the ledger of distributed funds on the blockchain. Each user is availed a Bitcoin wallet, a private and public key which all act in sync, Bitcoin wallet doesn't store the Bitcoin but only recording transactions and balance. The public key is 34 long alphanumeric and can be seen by anyone and though related to the private key,the 64 alphanumerics should remain personal as it acts as a digital signature when issuing transactions. Such an innovative approach to Bitcoin transactions makes Bitcoin transparent and secure to transact to both public who can access information by tracking the public key which provides all transactions but also the private key which safeguards the user's information. However, technologydoesn't hack-proof, many users have lost colossal sums of Bitcoin to hackers, the 2014 Mt.Gox case which lost an approximate \$470million worth of Bitcoin deflating prices by $50 \%$, to date Bitcoin has incurred an accumulated loss of more than 4billion dollars creating unease within governments and financial regulators resulting in stringent regulations in countries like Russia or total ban in the case of China.

Figure-4: Bitcoin transaction

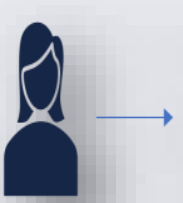

Sender

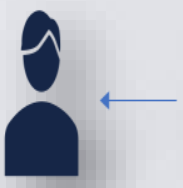

Receiver

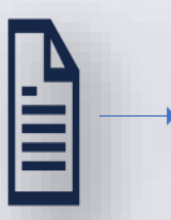

Bitcoin

Amount
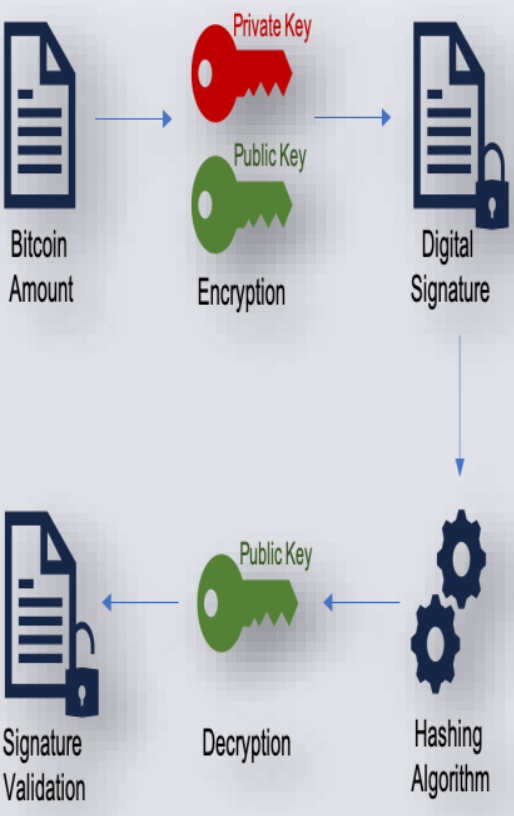

[4].www.coincover.com/post/how-are-Bitcoins-tradedand-what-is-a-wallet-signature

\section{IV.CONCLUSION:}

Acceptance, yet the biggest character cryptocurrencies and in particular Bitcoin has failed to achieve as currency volatility and extreme unpredictability defines Bitcoin creating a cloud of negative perceptions from the future users but also harsh regulation policies.

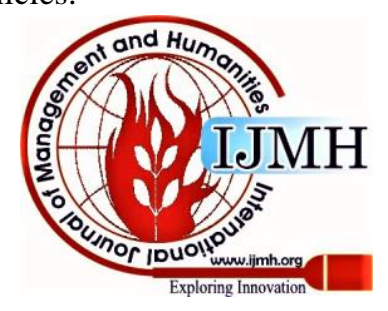


The current traditional system is based on fiat or in a few cases the gold standard, regulators can be able to predict inflation, recession and "overheating "of an economy through regulating the supply and demand of currency, this is not the case of Bitcoin which theoretically lacks any possibility of financial regulation as its value is based on artificial scarcity controlled by mathematical algorithms. However, financial regulators are not ignorant of solutions crypto-currency in particular Bitcoin offers in overhauling an outdated traditional currency defined by money laundering, high transactional fees,and incompatibility with the current technology which is based on online payment. "Libra" a currency developed by Facebook a USA based company is attempting to create a cryptocurrency dwarfing Bitcoin based on not a competition but initial collaboration with not only existing regulators of all currency; LibraUSD or $\approx \mathrm{USD}$, LibraEUR or $\approx \mathrm{EUR}$, LibraGBP or $\approx \mathrm{GBP}$, LibraSGD or $\approx$ SGD) but also partnership with intermediaries such as PayPal and MasterCard who have since distanced themselves from Libra. DC/EP, China's presumed digital currency also based on blockchain is being tested run in a few selected banks in anticipation of the 2022 Olympics but a possible merger with the upcoming 5G simcards. Ironically, Jamie Damion CEO JP Morgan and Stanley one of blockchain fierce critics launched the JPM Coin based on blockchain technology alongside the fiat system, and though currently a prototype in a few institutions, it's predicted to be used by businesses in the future.

Figure-5:Total Market Capitalization of Crypto-currency

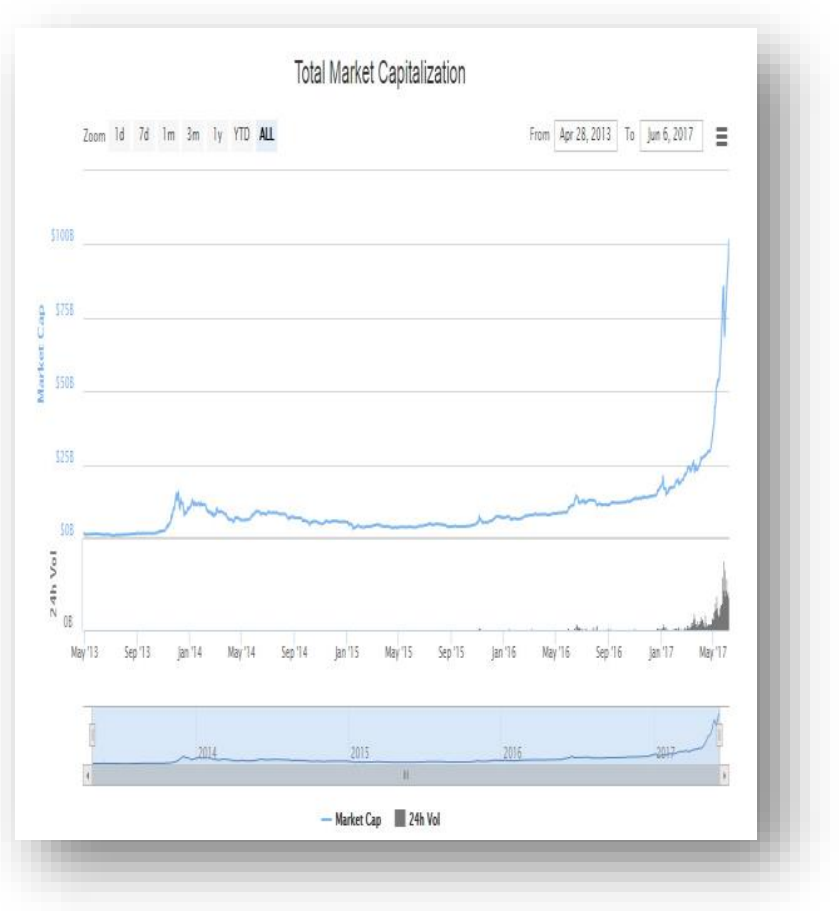

\section{[5]. Cryptomining-blog.com/tag/btc-market- capitalization/}

\section{REFERENCES:}

1. www.researchgate.net/figure/Bitcoin-mixingexample_fig1_322914722

2. www.medium.com/@liamzebedee/3-essential-takeaways-from-themit-microsoft-Bitcoin-talk-54a4cd71a702\#.17zeppbil

3. www.vamos.ee/easy-trade/limited-supply-of-Bitcoin-ethereum-mh/
4. www.coincover.com/post/how-are-Bitcoins-traded-and-what-is-awallet-signature

5. www.cryptomining-blog.com/tag/btc-market-capitalization/

\section{AUTHORS PROFILE}

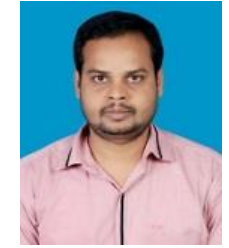

Dr. G. Taviti Naidu, is a doctorate in management from the Department of commerce \& management studies of Andhra University. I am qualified for NET and SET for lectureship. Presently, I am a Post Doctoral Fellow from ICSSR. I have authored and published research papers in reputed journals. Also, presented my papers in the prestigious institutes like IIM-A, IIM-I, Andhra University. My research interests are data analytics.

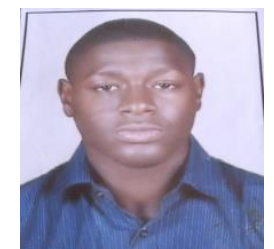

Muyinda Gerald Dhatemwa, is an African student born in Uganda, studying an MBA majoring in Finance at the prestigious university of Andhra, India. Born 26 years ago in a family of five to Mr.Godfrey Dhatemwa and Mrs. Suzan Kisakye, masters of in both their professional fields i.e. Economist and Principal secretary, who have inspired me to explore and achieve more not only academically but life as a whole.Having majored in Marketing as an undergraduate degree, I sought out both a challenge and companion field which could not only grow me as a scholar but also a practical and dynamic field to elevate my personal company in Uganda, alongside the guidance and wisdom of my senior professor Dr. Naidu a leading expert and scholar in the Research field at Andhra University

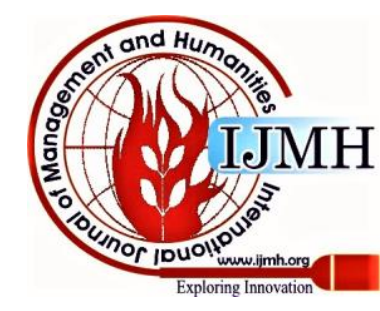

